\title{
Phase-Controlled Polarization Modulators
}

\author{
David T. Chuss, ${ }^{a}$ Edward J. Wollack, ${ }^{a}$ G. Novak, ${ }^{b}$ Giampaolo Pisano, ${ }^{c}$ J.R. Eimer, ${ }^{d}$ S.H. \\ Moseley, ${ }^{a}$ M. Krejny, ${ }^{e}$ K. U-Yen ${ }^{a}$ \\ ${ }^{a}$ NASA Goddard Space Flight Center, Observational Cosmology Laboratory, Code 665; \\ Greenbelt, MD, USA; \\ ${ }^{b}$ Northwestern University, Evanston, IL, USA \\ 'The University of Manchester, Manchester, UK \\ ${ }^{d}$ The Johns Hopkins University, Department of Physics and Astronomy, Baltimore, MD, USA \\ ${ }^{e} \mathrm{BAE}$ Systems, Columbia, MD, USA
}

\begin{abstract}
We report technology development of millimeter/submillimeter polarization modulators that operate by introducing a variable, controlled phase delay between two orthogonal polarization states. The variable-delay polarization modulator (VPM) operates via the introduction of a variable phase deiay between two linear orthogonal polarization states, resulting in a variable mapping of a single linear polarization into a combination of that Stokes parameter and circular (Stokes V) polarization. Characterization of a prototype VPM is presented at 350 and 3000 microns. We also describe a modulator in which a variable phase delay is introduced between right- and left- circular polarization states. In this architecture, linear polarization is fully modulated. Each of these devices consists of a polarization diplexer parallel to and in front of a movable mirror. Modulation involves sub-wavelength translations of the mirror that change the magnitude of the phase delay.
\end{abstract}

Keywords: Polarization Modulation, Astronomical Polarimetry

\section{INTRODUCTION}

Polarization modulation is a key element of many astronomical polarization systems. By systematically changing the polarization state to which an instrument is sensitive, it is possible to separate the polarized signal from a source and the associated unpolarized component that is often larger in magnitude and sometimes time variable. Specifically, polarization modulation can help to mitigate certain classes of systematic effects such as those associated with beam shape and, depending on the details of the system design, instrumental polarization. If performed rapidly, polarization modulation can also be utilized to mitigate $1 / f$ effects associated with the unpolarized component of the background. ${ }^{1}$

Polarization modulation can be considered ideal for cases in which the total polarization is not altered by the transformation process. That is, in terms of the Stokes parameters $(Q, U, V)$,

$$
P^{2} \equiv Q^{2}+U^{2}+V^{2}=\text { constant. }
$$

This condition can be visualized as the surface of a sphere of radius $P$ (the Poincaré Sphere) in a space having axes $Q, U$, and $V$. In the Jones matrix formalism, modulation operations are represented by unitary $2 \times 2$ complex matrices; in the homomorphically-equivalent Mueller formalism, ${ }^{2}$ they are represented by $4 \times 4$ orthogonal matrices.

Any rotation on the Poincaré Sphere can be generated by introducing a phase delay between two orthogonal polarizations. The two polarization states define the axis of rotation through the center of the sphere (orthogonal polarization states lie on opposite ends of a sphere diameter), and the magnitude of the phase delay is equal to the magnitude of the rotation on the Poincaré Sphere. ${ }^{3}$

Further author information: (Send correspondence to D.T.C.)

D.T.C.: E-mail: David.T.Chuss@nasa.gov, Telephone: 3012861858 
A popular polarization modulator that utilizes this concept is the rotating wave-plate. The wave-plate transforms the polarization by introducing a fixed delay between orthogonal linear polarizations. Modulation occurs through rotation of the wave-plate that changes the basis in which the delay is introduced. Half-wave plates, for which the delay is fixed at $\pi$, have been employed to measure linear polarization in the far-infrared and submillimeter. ${ }^{4}$ Recently, multi-layer versions have been produced that provide broad-band modulation for various astronomical polarimeters..$^{5-8}$ Reflective versions have also been implemented. ${ }^{9}$ Quarter-wave plates, for which the phase delay is fixed at $\pi / 2$, can be used for the measurement of circular polarization.

Alternatively, polarization modulation can be achieved by fixing the basis of phase separation and modulating the introduced phase. In this paper, we describe two architectures that utilize this approach for free space modulators. The first, the Variable-delay Polarization Modulator (VPM), operates through the introduction of a variable phase delay between two orthogonal linear polarizations resulting in a modulation between linear and circular polarization. The second modulator is the Translational Polarization Rotator (TPR). This device operates through the introduction of a variable phase delay between two orthogonal circular polarization states resulting in a modulation of Stokes $Q$ and $U$.

Dispersion effects in these phase-controlled polarization modulators are manifest differently than in waveplates. Specifically, because the signal spreading due to dispersion is in the same direction as the modulation on the Poincaré Sphere, the output signals as a function of phase separation for these modulators is the Fourier transform of the polarization spectrum for the modulated Stokes parameters. In addition, these modulators require small linear motions rather than the rotational motion required for wave-plates; this may be an advantage for space-based polarimetry.

\section{THE VARIABLE-DELAY POLARIZATION MODULATOR (VPM)}

\subsection{Operational Principle}

Figure 1A illustrates the basic concept of the VPM. The derice consists of a polarizer (wire grid) positioned in front of and parallel to a movable mirror. One linear polarization refiects off the front of the polarizer; the orthogonal polarization component passes through the grid, reflects off of the mirror and passes through the grid, and recombines with the first polarization. The introduced phase delay is related to the grid-mirror separation and can be varied to modulate the signal. The Mueller matrix representation is shown in Figure 1B. Assuming the wires are oriented in the $U$-direction, the modulation can be represented by the path on the Poincaré Sphere as depicted in Figure 1C.
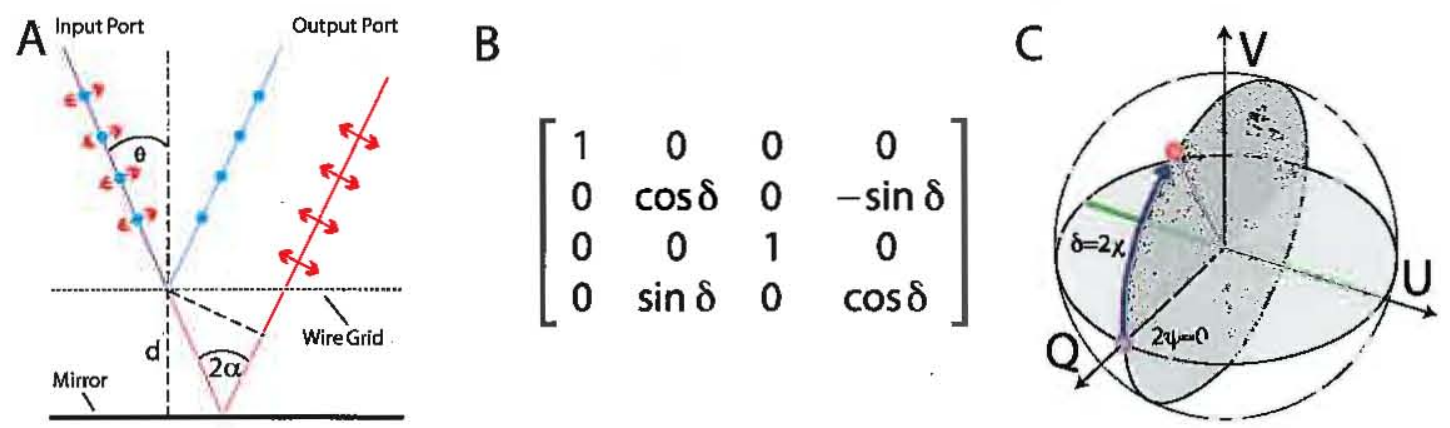

Figure 1. The V'PM consists of a wire grid polarizer positioned in front of and parallel to a movable mirror (A) and can be ideally represented by an orthogonal Mueller matrix (B). The polarization transfer function of the VPM can be represented by rotations on the Poincaré Sphere in the $Q-V$ plane. (C) In this case, the wires of the polarizer are parallel to the $U$-direction. 


\subsection{Implementation}

VPMs have been used in the Hertz/VPM instrument. ${ }^{10}$ In this case, a pair of VPMs was implemented, the first having wires oriented at $22.5^{\circ}$ with respect to the second. In this architecture, the VPMs are polarization svitches: the first switches between $U$ and $Q$ with the introduction of a $\pi$ phase delay, and the second uses a $\pi$ phase delay to switch between $\pm U$ or $\pm Q$, depending on the setting of the first modulator.

When the VPM is used in an instrument having detectors that are sensitive to linear polarization that is oriented at $45^{\circ}$ with respect to the VPM polarizer, the polarization transfer function is

$$
Q_{\text {detector }}=Q_{s k y} \cos \delta+V_{s k y} \sin \delta .
$$

Here, $\delta$ is the phase delay introduced by the VPM and all Stokes parameters are given with respect to the instrument reference frame.

The VPM is currently being implemented in two experiments designed to measure the polarization of the cosmic microwave background to search for the polarized signature of inflation. The small linear throws required and the reflective operation of the VPM allow modulators to be placed far forward in the optical systems of relatively low-resolution CNIB polarimeter. The forward placement of modulators that can be rapidly modulated enables the measurement of the CMIB polarization on large scales. In addition, since residuals of the polarization modulation are in Stokes $V$ (See Eq. 2), cross-polarization does not mix $Q$ and $U .^{11}$

The PIPER ${ }^{12}$ and CLASS ${ }^{13}$ cosmic microwave background polarimeters are each using VPMs in this configuration. They each employ large VPMs at the front of the optics in order to rapidly modulate polarization prior to the introduction of instrumental polarization by the optics. Because a VPMI-based polarimeter measures one linear Stokes parameter at a time, PIPER employs two telescopes: one dedicated to measuring Stokes $Q$ and one for Stokes $U$ in the instrument frame. CLASS employs an instrument rotator to completely measure linear polarization.

\subsection{Measurement}

It is critical to understand the relationship between the grid-mirror separation distance, $d$, and the introduced phese, $\delta$. If one considers only the geometric path difference,

$$
\delta=\frac{4 \pi d}{\lambda} \cos \theta .
$$

Here, $\lambda$ is the wavelength and $\theta$ is the angle of incidence of the radiation on the VPM. This expression is true in the long wavelength limit, i.e. when $\lambda \gg a$, where $a$ is the diameter of the wires of the polarizing grid. If this is not true, the reactance of the grid wires must be taken into account. In this case, a transmission line model can be used to model the transfer function of the VPMI. Figure 2 illustrates this. ${ }^{14}$ In Fig. 2A, the VPM response is shown for $a / \lambda=0.036$. For this case, the expression in Equation 3 fails. As one increases the wavelength, one find that the response of the VPMI approaches that which is consistent with Equation 3 (See Fig. 2B). In each case, the incident signal is $u \equiv U / I=1$. In Figure 2B, the dashed line is the model consistent with Equation 3.

In conjunction with the development of the VPM, rew metrology techniques have also been developed. Figure 3 illustrates the results of a method in which a vector network analyzer (VNA) is coupled to a free-space quasioptical setup via a feedhorn. A secondary calibration technique is applied in which the device under test is translated and measured multiple times. ${ }^{15}$ The differential phase information is used to extract the instrument response from the device under test. Figure 3 shows the result of applying this technique to a VPM that used the $50 \mathrm{~cm}$ diameter free standing polarizer described in Voellmer et al. ${ }^{16}$ (A) and the comparison of these data to a transmission line model for the VPM.

The VPM is closely related in its operational principle to the Martin-Puplett interferometer ${ }^{17}$ that has been most commonly used for far-infrared and submillimeter spectroscopy. Though it differs in implementation than the VPMs described here, the Martin-Puplett interferometer introduces a variable phase delay between two orthogonal linear polarizations. ${ }^{18}$ The PIXIE mission, ${ }^{19}$ a proposed spectropolarimeter for measuring the polarization of the cosmic microwave background, utilizes a Martin-Puplett interferometer for polarization modulation, measuring the polarized spectrum of the sky across the millimeter and submillimeter parts of the spectrum. 

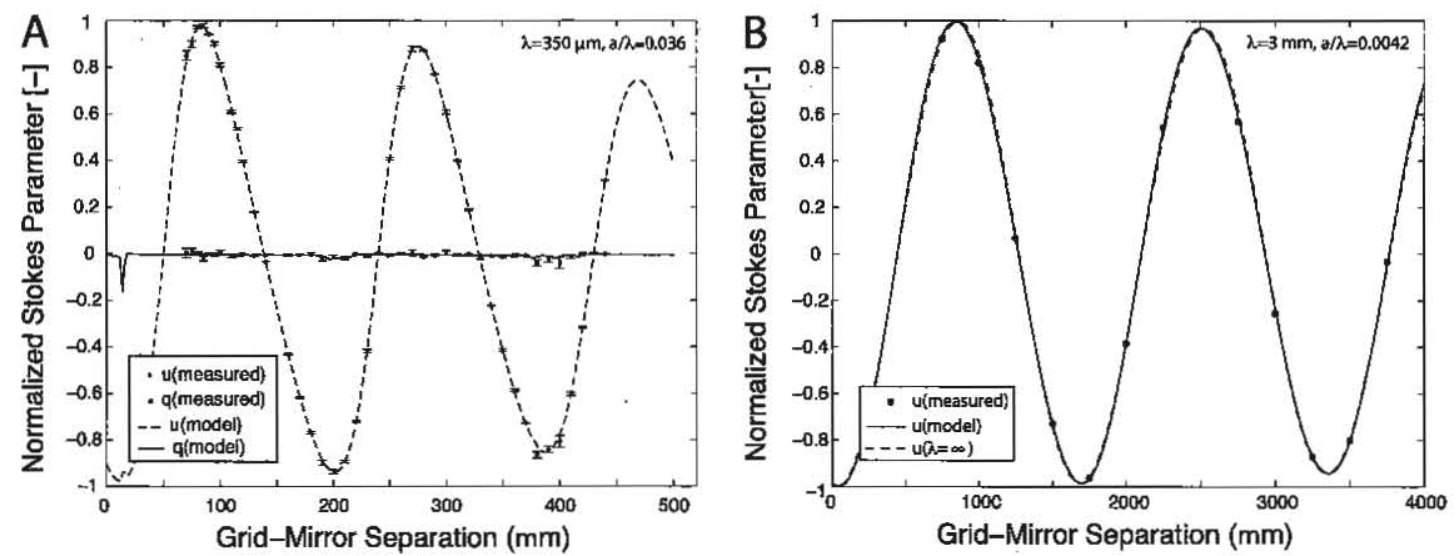

Figure 2. The polarization response of a VPM with input $q=1$ polarization at $350 \mu \mathrm{m}$ (A) and $3000 \mu \mathrm{m}$ (B) has been measured. ${ }^{14}$ These authors have found the VPM response to be well-fit using a transmission line model. The polarizing grid used had wire radius $a=12.5 \mu \mathrm{m}$ and $67 \mu \mathrm{m}$ wire spacing.
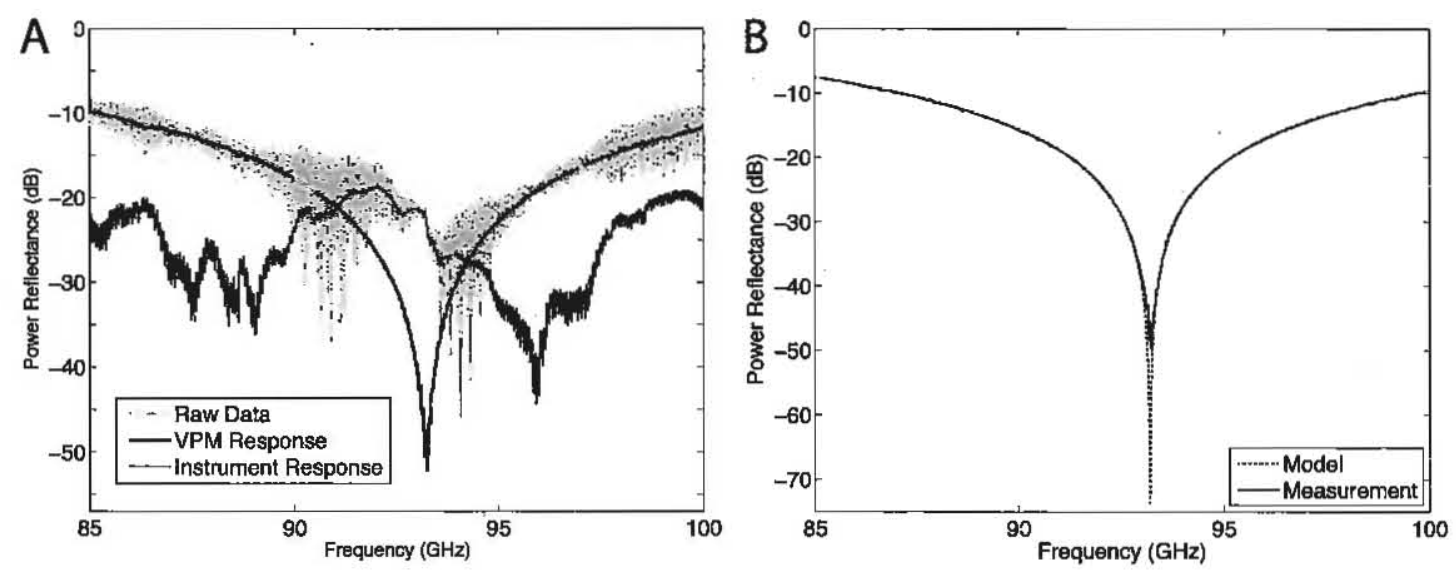

Figure 3. The polarization conversion of a VPM has been measured using a novel quasi optical vector network analyzer technique. ${ }^{15}$ The measurement was made using a large (50 cm aperture) polarizing grid with wire $67 \mu \mathrm{m}$ wire diameter and $200 \mu \mathrm{m}$ wire spacing. ${ }^{16}$ The separation of the raw data into the instrument and VPM responses is shown in (A). The extracted signal is compared to a VPM transmission model in (B). 


\section{THE TRANSLATIONAL POLARIZATION ROTATOR (TPR)}

\subsection{Operational Principle}

The TPR is related to the VPM; however, instead of using a linear basis for the phase introduction, the TPR introduces a variable phase delay between circular polarization states. This can be accomplished by using a circular polarizer in front of and paraliel to a movable mirror as shown in Figure 4 (A). Since the axis of phase separation is now $\pm V$, the modulation is represented by a circle in the $Q-U$ plane (See Fig. 4(B)). The Mueller matrix corresponding to this modulation topology is also shown in Figure 4(C).
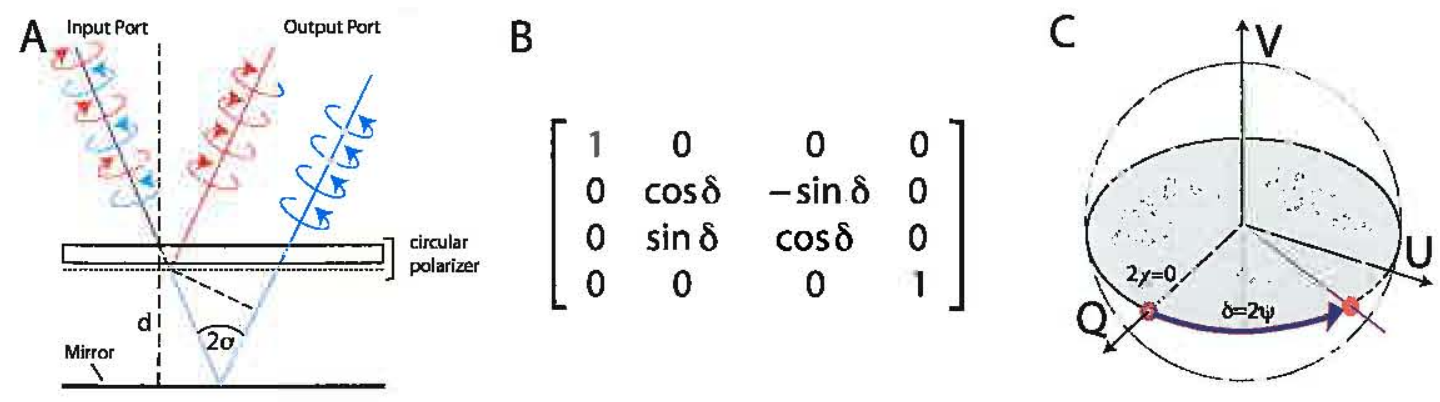

Figure 4. The TPR consists of a circular polarizer placed in front of and parallel to a movable reflector. (A) Its Mueller matrix is orthogonal and mixes $Q$ and $U(\mathrm{~B})$; modulation is represented by rotations in the $Q-U$ plane.

The transfer function for the TPR is

$$
Q_{\text {detector }}=Q_{3 k y} \cos \delta+U_{s k y} \sin \delta .
$$

\subsection{Implementation}

The key component for a TPR is the circular polarizer-e.g. a device that reflects right-handed circular polarization while transmitting its left-handed counterpart. Chuss et al. ${ }^{20}$ have synthesized a circular polarizer out of a meta.i mesh quarter-wave plate ${ }^{21}$ placed in front of a wire grid polarizer such that the wires are oriented at $45^{\circ}$ with respect to the fast (or slow) axis of the wave-plate. Preliminary measurements have been reported elsewhere and verify the basic principle. ${ }^{20}$

A key feature of the TPR is that it can fully modulate linear polarization with small, linear motions. In particular, for future large-aperture far-infrared space telescopes, the TPR can enable the addition of polarimetric capability to a photometric system with low cost and risk.

\section{SUMMARY}

We have described recent work on phase-controlled polarization modulators. This class of devices has potential applications in astronomical polarimetry. The VPM, which operates by introducing a variable phase delay between linear polarization states, is currently being employed in large angular scale suborbital CMB experiments. The TPR is a potential enabling technologv for polarimeters operating on future large aperture space-borne farinfrared telescopes. Further technological maturity of the TPR will depend on development of broad-band circuiar polarizers.

\section{REFERENCES}

[1] Brown, M. L., Challinor, A., North, C. E., Johnson, B. R., O'Dea, D., and Sutton, D., "Impact of modulation on CMB B-mode polarization experiments," Monthly Notices of the Royal Astronomical Society 397, 634656 (Aug. 2009).

[2] Sternberg, S., [Group Theory and Physics], Cambridge (1994).

[3] Tinbergen, J., [Astronomical Polarimetry], Cambridge (1996). 
[4] Hildebrand, R. H., Davidson, J. A., Dotson, J. L., Dowell, C. D., Novak, G., and Vaillancourt, J. E., "A Primer on Far-Infrared Polarimetry," Publications of the Astronomical Society of the Pacific 112, 1215-1235 (Sept. 2000).

[5] Savini, G., Pisano, G., and Ade, P. A. R., "Achromatic half-wave plate for submillimeter instruments in cosmic microwave background astronomy: modeling and simulation," Applied Optics IP 45, 8907 (Dec. 2006).

[6] Pisano, G., Savini, G., Ade, P. A. R., Haynes, V., and Gear, W. K., "Achromatic half-wave plate for submillimeter instruments in cosmic microwave background astronomy: experimental characterization," Applied Optics IP 45, 6982 (Sept. 2006).

[7] Savini, G., Ade, P. A. R., House, J., Pisano, G., Haynes, V., and Bastien, P., "Recovering the frequency dependent modulation function of the achromatic half-wave plate for POL-2: the SCUBA-2 polarimeter," Applied Optics 48, 2006 (Apr. 2009).

[8] Bryan, S. A., Montroy; T. E., and Ruhl, J. E., "Modeling dielectric half-wave plates for cosmic microwave background polarimetry using a Muelier matrix formalism," Applied Optics 49, 6313-6323 (Nov. 2010).

[9] Siringo, G., Kreysa, E., Kovács, A., Menten, K. M., and Forbrich, J., "Beginning of operation on APEX of the polarimeter for the Large APEX Bolometer Camera (LABOCA)," 7741 (July 2010).

[10] Krejny, M., Chuss, D., D'Aubigny, C. D., Golish, D., Houde, M., Hui, H., Kulesa, C., Loewenstein, R. F., Moseley, S. H., Novak, G., Voellmer, G., Walker, C., and Wollack, E., "The Hertz/VPM polarimeter: design and first light observations," Applied Optics 47, 4429 (Aug. 2008).

[11] Chuss, D. T., Ade, P. A. R., Benford, D. J., Bennett, C. L., Dotson, J. L., Eimer, J. R., Fixsen, D. J., Halpern, M., Hilton, G., Hinderks, J., Hinshaw, G., Irwin, K., Jackson, M. L., Jah, M. A., Jethava, N., Jhabvala, C., Kogut, A. J., Lowe, L., McCullagh, N., Miller, T., Mirel, P., Moseley, S. H., Rodriguez, S., Rostem, K., Sharp, E., Staguhn, J. G., Tucker, C. E., Voelimer, G. M.; Wollack, E. J., and Zeng, L., “The Primordial Inflation Polarization Explorer (PIPER)," in [Society of Photo-Optical Instrumentation Engineers (SPIE) Conference Series], Society of Photo-Optical Instrumentation Engineers (SPIE) Conference Series 7741 (July 2010).

[12] Kogut, A., Ade, P., Benford, D., Bennett, C., Chuss, D., Dotson, J., Eimer, J., Fixsen, D., Halpern, M., Hilton, G., Hinderks, J., Hinshaw, G., Irwin, K., Jhabvala, C., Johnson, B., Lazear, J., Lowe, L., Miller, T., Mirel, P., Moseley, S. H., Rodriguez, S., Tucker, C. E., Sharp, E., Staguhn, J., Weston, A., and Wollack, E., "The Primordial Inflation Polarization Explorer (PIPER)," Proc. SPIE 8452 (2012).

[13] Eimer, J., Bennett, C., Chuss, D. T., Marriage, T., Wollack, E., and Zeng, L., "The Cosmology Large Angular Scale Surveyor (CLASS): $40 \mathrm{GHz}$ optical design," Proc. SPIE 8452 (2012).

[14] Chuss, D., Wollack, E., Henry, R., Hui, H., Juarez, A., Krejny, M., Moseley, H., and Novak, G., "Properties of a variable-delay polarization modulator," Applied Optics 51, 197-208 (Jan. 2012).

[15] Eimer, J. R., Bennett, C. L., Chuss, D. T., and Wollack, E. J., "Note: Vector reflectometry in a beam waveguide," Review of Scientific Instruments 82(8), 086101 (2011).

[16] Voellmer, G. M., Bennett, C., Chuss, D. T., Eimer, J., Hui, H., Moseley, S. H., Novak, G., Wollack, E. J., and Zeng, L., "A large free-standing wire grid for microwave variable-delay polarization modulation," Ground-based and Airborne Instrumentation for Astronomy II. Edited by McLean 7014, 75 (Aug. 2008).

[17] Martin, D. and Puplett, E., "Polarised interferometric spectrometry for the millimetre and submillimetre spectrum," Infrared Physics 10, 105-109 (1970).

[18] Chuss, D. T., Wollack, E. J., Moseley, S. H., and Novak, G., "Interferometric polarization control," Applied Optics 45, 5107 (July 2006).

[19] Kogut, A., Fixsen, D. J., Chuss, D. T., Dotson, J., Dwek, E., Halpern, M., Hinshaw, G. F., Meyer, S. M., Moseley, S. H., Seiffert, M. D., Spergel, D. N., and Woliack, E. J., "The Primordial Inflation Explorer (PIXIE): a nulling polarimeter for cosmic microwave background observations," Journal of Cosmology and Astroparticle Physics 7, 25 (July 2011).

[20] Chuss, D. T., Wollack, E. J., Pisano, G., Ackiss, S., U-Yen, K., and Ng, M. w., "A Translational Polarization Rotator," ArXiv e-prints (June 2012).

[21] Pisano, G., Savini, G., Ade, P. A. R., and Haynes, V., "Metal-mesh achromatic half-wave plate for use at submillimeter wavelengths," Applied Optics 47, 6251 (Nov. 2008). 